\title{
Integrity of cerebral white matter in type 1 diabetes. Reply to Wessels AM [letter]
}

\author{
K. Weinger • A. M. Jacobson • G. Musen • I. K. Lyoo • \\ C. M. Ryan • P. F. Renshaw
}

Received: 1 May 2008 /Accepted: 6 May 2008 / Published online: 10 June 2008

(C) Springer-Verlag 2008

Keywords Brain imaging - Cognition · Type 1 diabetes . White matter hyperintensities $\cdot$ WMH

\section{Abbreviation}

WMHs white matter hyperintensities

To the Editor: We appreciate the valuable comments of $\mathrm{Dr}$ Wessels [1] on our recent article in Diabetologia [2]. We specifically sought to study the impact of white matter hyperintensities (WMHs) on brain structure in relatively young patients (age $32 \pm 4$ years) with diabetes of long duration ( $20 \pm 4$ years) (mean $\pm \mathrm{SD}$ ). As Wessels noted, our large sample of patients did not differ from non-diabetic controls in terms of severity of white matter lesions. Indeed,

K. Weinger $(\bowtie) \cdot$ A. M. Jacobson $\cdot$ G. Musen

Behavioral and Mental Health Research, Joslin Diabetes Center,

One Joslin Place, Suite 350,

Boston, MA 02459, USA

e-mail: katie.weinger@joslin.harvard.edu

K. Weinger · A. M. Jacobson · G. Musen • P. F. Renshaw Department of Psychiatry, Harvard Medical School,

Boston, MA, USA

I. K. Lyoo • P. F. Renshaw

Brain Imaging Center, McLean Hospital,

Belmont, MA, USA

I. K. Lyoo

Seoul National University College of Medicine and Hospital,

Seoul, Korea

C. M. Ryan

Department of Psychiatry,

University of Pittsburgh School of Medicine,

Pittsburgh, PA, USA our results are similar to those of Brands et al. [3], who studied cognition and WMHs in older type 1 diabetic adults with disease of longer duration associated with more complications. Taken together, these studies suggest that the incidence of WMHs in type 1 diabetes is not likely to be high. Using voxel-based morphometry, we have also found that, compared with non-diabetic controls, patients with type 1 diabetes had lower grey matter density and this was associated with both higher $\mathrm{HbA}_{1 \mathrm{c}}$ levels and an increased number of severe hypoglycaemic events [4]. This finding suggests that more sensitive measures of brain structure would reveal changes induced by type 1 diabetes.

Wessels notes that type 1 diabetes leads to changes in cognition, which may be mediated by hyperglycaemia, hypoglycaemia and/or vascular disease. The literature on these points is far from conclusive. We observed only small differences in cognition when comparing type 1 diabetic patients to non-diabetic controls [2]. Our results are consistent with a meta-analysis that found modest effects of type 1 diabetes on cognition, characterised by slowing of mental processing and diminished mental flexibility [5]. Furthermore, our cognitive data were consistent with recent results from an 18 year longitudinal study of type 1 diabetic patients enrolled in the Diabetes Control and Complications Trial [6], which found only minimal changes in psychomotor efficiency and motor speed over time among those with the highest $\mathrm{HbA}_{1 \mathrm{c}}$ levels.

Wessels' suggestion that we examine our WMH findings in relation to total white matter volume measurements in patients with and without retinopathy is of interest and would be a useful complementary design. Our goal is to utilise multiple methods of assessing the grey and white matter compartments of the brain, to develop a more precise understanding of the 'signature' of type 1 diabetes. 
We have some reservations about considering the categorical rating method as being a less sensitive method than a fully quantitative volumetric analysis, as Wessels suggests [1]. To our understanding, categorical and quantitative assessments of WMH lesions have different purposes, and both methods have strengths and weaknesses.

Volumetric analysis, using both manual segmentation methods [7] and recent automated procedures [8] to measure WMH volumes, has several methodological limitations, including inter-rater reliability, partial volume effects (especially problematic in the T2-weighted images, the slice thickness of which is usually $>3 \mathrm{~mm}$ ), intensity distortion caused by magnetic field inhomogeneity and issues in processing low-quality MR images such as those obscured by motion artefacts. Some of these factors can be efficiently taken into consideration when the images are evaluated by neuroradiologists. Furthermore, we note that automatic and semiautomatic volumetric procedures have generally been validated using visual inspection scales or clinical variables as reference standards [8], which also introduces a subjective component. On a related note, the Fazekas classification system we adopted in our analysis as our primary scoring system has been histopathologically validated [9].

Although controversy exists [10], WMHs in the brain are likely to have different effects on neurological and psychiatric symptomatology according to their specific locations [11]. The visual inspection scale that we used also provides information regarding the locations of the lesions.

In conclusion, our study examined WMHs using current standard imaging techniques in a large sample of diabetic patients and matched non-diabetic controls, and the results add to our knowledge of the relationship, or lack thereof, between these lesions and diabetes or cognitive deficits. Minimal white matter lesions were found, and these were not associated with diabetes or cognition. However, newer, more sensitive imaging techniques may find differences in brain structure, metabolism or function between those with and without diabetes.

\section{References}

1. Wessels AM (2008) Integrity of cerebral white matter in type 1 diabetes. Diabetologia DOI 10.1007/s00125-008-1011-2

2. Weinger K, Jacobson AM, Musen G et al (2008) The effects of type 1 diabetes on cerebral white matter. Diabetologia 51:417-425

3. Brands AM, Kessels RP, Hoogma RP et al (2006) Cognitive performance, psychological well-being, and brain magnetic resonance imaging in older patients with type 1 diabetes. Diabetes 55:1800-1806

4. Musen G, Simonson DC, Bolo NR et al (2008) Regional brain activation during hypoglycemia in type 1 diabetes. J Clin Endocrinol Metab 93:1450-1457

5. Brands AM, Biessels GJ, de Haan EH, Kappelle LJ, Kessels RP (2005) The effects of type 1 diabetes on cognitive performance: a meta-analysis. Diabetes Care 28:726-735

6. Jacobson AM, Musen G, Ryan CM et al (2007) Long-term effect of diabetes and its treatment on cognitive function. N Engl J Med 356:1842-1852

7. DeCarli C, Murphy DG, Tranh M et al (1995) The effect of white matter hyperintensity volume on brain structure, cognitive performance, and cerebral metabolism of glucose in 51 healthy adults. Neurology 45:2077-2084

8. Maillard P, Delcroix N, Crivello F et al (2008) An automated procedure for the assessment of white matter hyperintensities by multispectral (T1, T2, PD) MRI and an evaluation of its betweencentre reproducibility based on two large community databases. Neuroradiology 50:31-42

9. Fazekas F, Kleinert R, Offenbacher H et al (1993) Pathologic correlates of incidental MRI white matter signal hyperintensities. Neurology 43:1683-1689

10. DeCarli C, Fletcher E, Ramey V, Harvey D, Jagust WJ (2005) Anatomical mapping of white matter hyperintensities (WMH): exploring the relationships between periventricular $\mathrm{WMH}$, deep WMH, and total WMH burden. Stroke 36:50-55

11. de Groot JC, de Leeuw FE, Oudkerk M, Hofman A, Jolles J, Breteler MM (2001) Cerebral white matter lesions and subjective cognitive dysfunction: the Rotterdam Scan Study. Neurology 56:1539-1545 